\title{
COMPROMETIMENTO DA SEXUALIDADE DE MULHERES COM CÂNCER DE MAMA
}

Ana Inêz Severo Varela ${ }^{1}$, Luciana Martins da Rosa², Natália Sebold², Ana Gabriela laverde², Amarildo Maçaneiro', Alacoque Lorenzini Erdmann ${ }^{3}$

Objetivo: identificar os obstáculos relacionados à sexualidade e à vida sexual de mulheres com câncer de mama. Metodologia: pesquisa descritiva, que incluiu dez mulheres submetidas à mastectomia ou quandrantectomia residentes no municipio de Florianópolis, selecionadas pelo método bola de neve. Dados obtidos por entrevistas semiestruturadas, realizadas entre março e maio de 2014, submetidos à análise de conteúdo e sustentados teoricamente por estudos afins. Resultados: os obstáculos encontrados envolvem o enfrentamento familiar e pessoal comprometidos e a alteração sexual e ginecológica. Conclusão: apesar do avanço científico e tecnológico, permanece a necessidade de cuidado integral e individualizado à mulher, aos companheiros e aos familiares.

Descritores: Sexualidade, Neoplasias da mama, Mastectomia, Enfermagem.

\section{COMMITMENT TO SEXUALITY OF WOMEN WITH BREAST CANCER}

Objective: Identify obstacles related to sexuality and sexual life of women with breast cancer. Methodology: descriptive study, which included ten women undergoing mastectomy or quandrantectomia, residents in Florianopolis, selected by snowball method. Data from semistructured interviews, conducted between March and May 2014 and submitted to content analysis and theoretically supported by related studies. Results: The obstacles encountered includes confrontation of family and personal commitment and sexual and gynecological change. Conclusion: Despite the scientific and technological advances remain the need for comprehensive care and individualized to women, to companion and family.

Descriptors: Sexuality, Breast neoplasms, Mastectomy, Nursing.

\section{COMPROMISO DE LA SEXUALIDAD DE LAS MUJERES CON CÁNCER DE MAMA}

Objetivo: Identificar los obstáculos relacionados con la sexualidad y la vida sexual de las mujeres con cáncer de mama. Metodología: estudio descriptivo, que incluía diez mujeres sometidas a mastectomía o quandrantectomia, residentes en Florianópolis, seleccionados por el método bola de nieve. Los datos recogidos por entrevistas semi-estructuradas, entre marzo y mayo de 2014, sometido a análisis de contenido y, en teoría apoyada por estudios relacionados. Resultados: Los obstáculos encontrados implican hacer frente familia y la evolución sexual y ginecológica personal y comprometido. Conclusión: A pesar de los avances científicos y tecnológicos continúa la necesidad de una atención integral e individualizada a las mujeres, compañeros y familiares.

Descriptores: Sexualidad, Neoplasias de la mama, Mastectomía, Enfermería. 


\section{INTRODUÇÃO}

O câncer de mama é o de maior incidência entre as mulheres e ocupa o segundo lugar no painel geral de incidência de câncer no Brasil. Apesar disso, as taxas de sobrevida apresentam-se com crescente elevação, graças às novas tecnologias de tratamento e para detecção precoce, especialmente nos países desenvolvidos. No ano de 2012, a incidência do câncer de mama no mundo foi 1.676.633. Para o Brasil, em 2016, são esperados 57.960 casos novos de câncer de mama ${ }^{(1,2)}$.

A mulher acometida pelo câncer de mama vivencia o impacto negativo que a doença e as terapêuticas ocasionam sobre as funções sistêmicas, mas, principalmente, sobre a imagem corporal, destacando-se o impacto do enfrentamento da mastectomia, mesmo para aquelas mulheres que realizam a reconstrução mamária. Assim, quanto menos mutiladoras forem as consequências da cirurgia, mais qualidade de vida a mulher poderá desfrutar (3).

As mamas estão fortemente ligadas à sexualidade e à feminilidade, quando alteradas pelo câncer e pelas terapêuticas de controle da doença, a sexualidade da mulher é afetada. Muitas mulheres precisam de ajuda para superar o trauma da doença e do tratamento, bem como para retomar, plenamente, a prática da sexualidade ${ }^{(4)}$. Portanto, é preciso que os profissionais conheçam as dificuldades que permeiam a sexualidade frente ao câncer de mama, a fim de melhorar a comunicação e o vínculo terapêutico para assegurar-se melhor assistência.

Assim, a alteração da estética da imagem corporal e da sexualidade são aspectos inerentes ao câncer de mama, sendo que, essas alterações necessitam ser consideradas continuamente na prática profissional da enfermagem, principalmente quando pensamos em prestar um cuidado integral e humanizado(b). Mas, ainda observa-se comunicação prejudicada entre os profissionais da área da saúde e as mulheres com câncer de mama(7).

Nesse contexto, questiona-se: quais são os obstáculos enfrentados pelas mulheres com diagnóstico de câncer de mama sobre a sexualidade e vida sexual? E estabelece-se o seguinte objetivo deste estudo: identificar os obstáculos relacionados à sexualidade e à vida sexual de mulheres com câncer de mama.

Esse objetivo foi firmado considerando que em outro estudo desenvolvido, que objetivou a identificação das estratégias adotadas por mulheres para minimizar os danos que as terapêuticas utilizadas para o controle do câncer de mama causam sobre a sexualidade e vida sexual, foram encontradas estratégias, mas em virtude do expressivo relato dos obstáculos, considerou-se importante esta divulgação.

\section{METODOLOGIA}

Trata-se de uma pesquisa descritiva aprovada eticamente, CAAE 26203413000000121.

Foram incluidas dez mulheres com câncer de mama, submetidas à mastectomia total ou quandrantectomia, com ou sem linfadenectomia axilar há três ou mais anos, em remissão da doença ou realizando hormonioterapia e residentes no município de Florianópolis. A seleção das mulheres foi pelo método bola de neve, que permite que os participantes do estudo indiquem outros participantes para serem incluídos na pesquisa. A primeira entrevista foi indicada por enfermeira especialista em enfermagem oncológica atuante em Florianópolis. $O$ número de mulheres incluídas no estudo foi definido pela saturação dos dados. A coleta dos dados ocorreu entre março e maio de 2014.

Para a coleta dos dados, foram realizadas entrevistas semiestruturadas. A entrevista somente foi realizada após concordância e assinatura do Termo de Consentimento Livre e Esclarecido. A pergunta que orientou o desenvolvimento do estudo foi: quais são os obstáculos que você ainda vivencia em virtude do diagnóstico de câncer de mama e das mudanças ocasionadas pelo tratamento sobre a sua sensualidade e vida sexual?

As entrevistas gravadas foram transcritas e submetidas à análise de conteúdo(8). A inferência foi sustentada por estudos afins às categorias temáticas. Para garantir o anonimato, cada participante do estudo escolheu um nome de uma flor para representar seus relatos.

\section{RESULTADOS}

As participantes do estudo totalizaram dez mulheres, sete foram submetidas à mastectomia total com linfadenectomia axilar, duas à quadrantectomia com linfadenectomia axilar e uma foi submetida a dois procedimentos, mastectomia total com esvaziamento axilar e quadrantectomia sem esvaziamento axilar (ambas as mamas); seis das entrevistadas convivem com o diagnóstico de câncer de mama há menos de dez anos e quatro há mais de dez anos. As categorias temáticas, resultantes da análise das comunicações foram: enfrentamento familiar comprometido, enfrentamento pessoal comprometido e alteração sexual e ginecológica. No quadro 1, apresentam-se as categorias, temas e unidades de significação (depoimentos das participantes que melhor expressam os temas por categoria temática). 
Quadro 1 - Obstáculos vivenciados por mulheres com câncer de mama, categorias temáticas, temas e unidades de significação. Florianópolis-SC, Brasil. 2014.

\section{CATEGORIAS}

Enfrentamento conjugal

comprometido

Enfrentamento pessoal comprometido

Depressão / tristeza

Dificuldades para falar

sobre sexo

Traumas psicológicos

Tratamento sentido como sofrimento

\section{UNIDADES DE SIGNIFICAÇÃO}

Era casada... Afetou muito... Marcou muito porque meu marido não me tocava. E isso pra mim foi muito difícil. Um homem que dormia comigo e que não estava conseguindo lidar com a situação. E eu tentei ajudar, tentei... Mas, depois eu fiquei pensando, não cabe a mim, sabe? Porque se pra ele precisa da mulher escultural, peituda, bunduda, a mulher gostosa, que todo mundo diz que a mulher brasileira é assim, o que eu posso fazer? O que eu posso fazer se não sou eu essa mulher? Dai eu acho que as coisas degringolaram um pouco. (Rosa Vermelha)

[...] quando eu tive câncer de mama, eu vivia com um senhor, aí quando eu descobri o câncer e ele saiu. [...] eu acho que como ele já era mais de idade, ele achou que o câncer ia pegar. Hoje eu me dou bem com ele, mas eu não tive mais nada, por causa desses problemas [...]. (Gérbera)

[...] me sentia desconfortável por causa da cicatriz. (Lírio)

Entrei em depressão, sai da depressão, entrei na depressão de novo, saí da depressão (risos), eu acredito que o quadro depressivo nunca vai deixar de existir. [...] E no meu caso, afetou a parte física e a emocional. E eu não consigo passar essa... Então, eu deixo a vida levar! (Margarida)

Imagina sem uma mama! E eu era acostumada a usar decote, e coisa... Bem à vontade... Depois passei a usar coisas que me deixam sufocada, pois, se me abaixava parecia que tava todo mundo te olhando... Ninguém notava que eu não tinha um seio, mas pra minha cabeça notavam [...] (Rosa)

Por causa do câncer, eu sofri muito com o tratamento e, sei lá, não quis arrumar ninguém naquela época, tava em função do tratamento, fui deixando, fui deixando... hoje eu fiquei traumatizada, eu não sei nem se eu consigo dilatar, não sei nem se eu consigo ter mais relação. (Gérbera)

Quando eu fiz a cirurgia, eu tive um bloqueio e eu não queria saber de hospital de jeito nenhum! (Rosa)

[...] sou mastectomizada e parece que isso separa, que isso assusta o homem. Então, para mim a vida sexual morreu. (Hortência)

O problema é que a gente fica seca, aí eu vi que até depois que terminou o tratamento, durante o tratamento a relação doía, a relação dói porque aquilo ali fica seco, ai eu usava aquele $\mathrm{KY}$, aquilo ardia. [...] mas, eu acho que quando terminasse a quimi..., [...] agora dá certo, vou voltar ao normal, eu vou ter lubrificação eu vou ter vontade de fazer sexo, mas não. Passaram os cinco anos do tamoxifeno e a coisa continuou igual e até achei que ficou mais dolorido. (Rosa Vermelha)

Minha relação sexual, [...]. afetou claro porque a gente fica com ressecamento vaginal, fica mais dolorido. Eu atualmente não tenho mais aquele tesão, isso acaba, e com essa situação minha de não haver penetração, dói só em tocar, não faço mais o preventivo[...] (Tulipa) 


\section{DISCUSSÃO}

O Enfrentamento Familiar Comprometido foi relatado pela maioria das mulheres entrevistadas. Os relatos mostraram maridos e companheiros despreparados para o enfrentamento do processo de adoecer e tratar o câncer junto à companheira. A autoestima e autoimagem dessas mulheres já fragilizadas e/ou prejudicadas pelo diagnóstico e efeitos do tratamento agressivo em seus corpos, agravaramse pelo fato de serem "rejeitadas" pelos companheiros.

Nesse sentido, o enfermeiro e os profissionais da saúde têm a possibilidade de atuar e ter papel fundamental para apoiar e ajudar a mulher e seu companheiro, identificandose os problemas e as necessidades. Porém, estudo afirma que a comunicação sobre sexualidade com os profissionais de saúde é descrita pelas mulheres com câncer de mama como prescritiva, com pouco espaço para diálogo e para a reflexão(9).

A sexualidade e sensualidade podem ser melhoradas por meio de redes de suporte, como grupos de apoio, para envolver e aproximar mais o companheiro da mulher com câncer de mama e contribuir para um maior entendimento das condições sociais e afetivas. O cuidado prestado às mulheres com câncer de mama deve ser de forma humanizada, individualizada e integral(5-7,10,11).

Muitos casais conseguem enfrentar o câncer e sair mais fortalecidos, porém muitos não conseguem. $O$ tipo de relacionamento anterior ao adoecimento também corrobora para essa condição. Nessas fases, a atenção interdisciplinar é extremamente relevante, pois as diversas abordagens podem ajudar a mulher, seu companheiro a compreenderem a nova situação de vida e a se tornarem resilientes, na busca de alternativas para um novo viver, com alegria e qualidade de vida.

O Enfrentamento Pessoal Comprometido foi encontrado em mais da metade das entrevistadas. Enfrentar o câncer de mama significa a elaboração de novas escolhas de cuidados, de um novo olhar sobre a vida e o modo de viver. Esse processo gera ou obriga adaptações, impulsionadas pela luta pela sobrevivência em busca da resiliência.

Estudo aponta que, para melhorar os sentimentos negativos que as mulheres têm com a chegada da doença, é necessário apoio familiar, do companheiro e dos profissionais, ajudando-a aos poucos a voltar ao seu papel ativo na sociedade. A participação em grupo de apoio com mulheres que vivenciam a mesma experiência e a reabilitação psicossocial deve ser incentivada. Devendo os serviços de saúde estar preparados para recebê-las, entendê-las, tirando suas dúvidas e dando um cuidado humanizado ${ }^{(12)}$.
Estudos comparativos com sobreviventes de câncer de mama observaram prejuízo na qualidade de vida geral e para os domínios físico e psicológico, em relação às mulheres sem exposição a qualquer doença crônica e, para os funcionamentos físico, de papéis, emocional, cognitivo e social em relação às mulheres da população em geral ${ }^{(13)}$. sendo que, o enfrentamento ineficaz frente à nova condição de vida colaboram com esse prejuízo.

A Alteração da imagem corporal também foi revelada por mais da metade das mulheres entrevistadas e trouxe consequências sobre a identidade da mulher, fortemente influenciada pelos aspectos culturais, sociais e familiares.

Nesse contexto, éimportanteressaltar que a enfermagem por meio do diálogo, da problematização, das vivências e das trocas de informações pode proporcionar o surgimento de novos aprendizados ${ }^{(14)}$, possibilitar à mulher compreender a doença e suas consequências e, mais uma vez, favorecer a resiliência e a sobrevivência, mesmo pós-diagnóstico de câncer.

Assim, diante dos relatos, observa-se a importância da avaliação de enfermagem e multiprofissional sobre os aspectos biopsicossociais e capacidade de aceitação como uma contribuição para que a mulher mantenha seu papel, apesar das mutilações vivenciadas e das mudanças no dia a dia pós-diagnóstico de câncer de mama.

A Alteração Sexual e Ginecológica foi encontrada quase que na totalidade dos relatos das mulheres incluidas neste estudo. A mudança da estética corporal causa inibição durante a relação sexual, e gera sentimentos de angústia, vergonha e constrangimento. A mastectomia altera a altopercepção e autoestima das mulheres, gera ansiedade e confusão, inclusive na decisão da reconstrução mamária. Somado a isto, a mulher ainda sofre os efeitos da quimioterapia, que altera a função sexual. Mulheres na pré-menopausa têm efeitos menores que as mulheres na pós-menopausa, principalmente, a longo prazo. $\mathrm{O}$ aconselhamento e a educação em saúde podem reduzir a ansiedade, trazendo segurança e melhores escolhas de cuidado ${ }^{(15,16)}$.

Os achados encontrados neste estudo assemelham-se aos encontrados em estudo qualitativo descritivo realizado em Brasilia/DF, que também evidenciou que as mulheres relatam tristeza, ansiedade, vergonha, exclusão e mutilações consequentes às terapêuticas ${ }^{(17)}$.

Por fim, registra-se como limite deste estudo o método bolo da neve, pois como nesse método as indicações dos participantes são consequentes às indicações das próprias participantes, esse aspecto pode ser considerado um limitador por outros pesquisadores. Como pesquisadoras 
deste estudo, a seleção segundo o método bola de neve permitiu seleção aleatória, assim foi percebida como um contribuidor

\section{CONCLUSÃO}

Os obstáculos encontrados nesta investigação mostram sua repercussão sobre o enfrentamento pessoal e familiar comprometidos, bem como causando alterações sobre a sexualidade e características e ginecológicas.

Os resultados reforçam a necessidade dos enfermeiros adotarem uma postura mais aberta às questões voltadas à sexualidade e à sensualidade, ao cuidado integral e individualizado à mulher, companheiros e familiares. Reforçam ainda a necessidade de orientação/educação à saúde das mulheres com câncer de mama, dos seus companheiros e da importância da atenção psicológica.

Nesse sentido, recomendam-se o uso dos diagnósticos de enfermagem e o estabelecimento de intervenções de enfermagem para atendimento das necessidades das mulheres com câncer de mama, em busca de uma melhor sexualidade e qualidade de vida, mesmo após o diagnóstico da doença.

\section{REFERÊNCIAS}

1. Ferlay J, Soerjomataram I, Dikshit R, Eser S, Mathers C. Rebelo M et al. International Journal of Cancer [Internet]. Cancer incidence and mortality worldwide: sources, methods and major patterns in GLOBOCAN 2012; 2014

2. Instituto Nacional de Câncer José Alencar Gomes da Silva. Estimativa 2016: incidência de câncer no Brasil. Rio de Janeiro: INCA; 2015.

3. Simeão SF AP, Landro ICR, De Conti MHS, Gatti MAN, Delgallo WD, De Vitta A. Qualidade de vida em grupos de mulheres acometidas de câncer de mama. Ciênc saúde coletiva [Internet]. 2013 mar; [acesso em 2017 fev 17] 18(3): 779-788. Disponivel em: http://www.scielo.br/scielo.php?script=sci_ arttextEpid=S1413-81232013000300024\&lng=enEnrm=iso

4. Ferreira SMA, Panobianco MS, Gozzo TO, Almeida AM. A sexualidade da mulher com câncer de mama: análise da produção científica de enfermagem. Texto Contexto Enferm [Internet]. 2013 jul-set; [acesso em 2017 fev 17]; 22(3): 835-42. Disponivel em: www.scielo.br/pdf/tce/v22n3/ v22n3a33.pdf

5. Santos DB, Santos MA, Vieira EM. Sexualidade e câncer de mama: uma revisão sistemática da literatura. Saúde Soc. São Paulo [Internet]. 2014 [acesso em 2016 nov 20]; 23(4):1342-55. Disponivel em: http://www.scielo. br/pdf/sausoc/v23n4/0104-1290-sausoc-23-4-1342.pdf

6. Verenhitach BD, Medeiros JN, Elias S, Nazário ACP. Câncer de mama e seus efeitos sobre a sexualidade: uma revisão sistemática sobre abordagem e tratamento. FEMINA [Internet]. 2014 jan-fev [acesso em 2016 ago 22]; 42(1), Disponivel em: http://files.bvs.br/upload/S/0100-7254/2014/v42nl/ a4806.pdf

7. Junqueira LCU, Vieira EM, Giami A, Santos MA. Análise da comunicação acerca da sexualidade, estabelecida pelas enfermeiras, com pacientes no contexto assistencial do câncer de mama. Interface (Botucatu) [Internet]. 2013 mar [acesso em 2016 ago 22]; 17(44): 89-101. Disponivelem: http://www. scielo.br/scielo.php?script=sci_arttextEpid=\$1414-32832013000100008

8. Bardin L. Análise de conteúdo. Lisboa: Editora 70; 2011.

9. Vieira EM, Santos DB, Santos MA, Giami A. Vivência da sexualidade após o câncer de mama: estudo qualitativo com mulheres em reabilitação Rev Latino-Am Enfermagem [Internet]. 2014 [acesso em 2016 nov 23]; 22(3):40814. Disponivel em: http://www.scielo.br/pdf/rlae/v22n3/pt_0104-1169rlae-22-03-00408.pdf
10. Rosa LM, Radunz V. Significado do câncer de mama na percepção da mulher: do sintoma ao tratamento. Rev Enferm UERJ. [Internet]. 2012 outdez [acesso em 2016 ago 22]; 4(20):445-50. Disponivel em: http://www. facenf.uerj.br/v20n4/v20n4a06.pdf

11. Alvares AS, Corrêa ACP. Arantes RB. Câncer de Mama e Colo do Útero: estrutura organizacional da prevenção na estratégia saúde da família. Enferm. Foco [Internet]. 2013 [acesso em 2016 ago 22]; 4(3/4): 153-6. Disponivel em: http://revista.portalcofen.gov.br/index.php/enfermagem/ article/view/538/221

12. Ribeiro VC. Portella SDC. Malheiro ES. Mulheres de meia idade e o enfrentamento do câncer de mama. Rev Cuid [Internet]. 2014 [acesso em 2016 nov 23]; 5(2): 799-805. Disponivel em: http://www.revistacuidarte.org/ index.php/cuidarte/article/view/120/265

13. Kluthcovsky ACGC, Urbanetz AAL. Qualidade de vida em pacientes sobreviventes de câncer de mama comparada à de mulheres saudáveis. Rev Bras Ginecol Obstet [Internet]. 2012 out [acesso em 2016 ago 22] 34(10):453-8. Disponivel em: http://www.scielo.br/scielo.php?script=sci_ arttext\&pid=S0100-72032012001000004

14. Santos GS, Teixeira MB. Diagnósticos de enfermagem frequentes em idosos residentes na área de abrangência de uma Estratégia Saúde da Familia. REFACS [Internet]. 2013 [acesso em 2016 ago 22]; 1(1):34-41. Disponivel em: http://www.uftm.edu.br/revistaeletronica/index.php/ refacs/article/view/605/424. 1

15. Yi M, Joung WJ, Park EY, Kwon EJ, Kim H, Seo JY. Decision Making Experience on Breast Reconstruction for Women with Breast Cancer.J Korean Acad Nurs [Internet]. 2016 dez [acesso em 2017 fev 17]; 46(6):894904. Disponivel em: https://www.ncbi.nlm.nih.gov/pubmed/28077836

16. Farthmann J, Hanjalic-Beck A, Veit J, Rautenberg B, Stickeler E, Erbes T, Földi M, Hasenburg A. The impact of chemotherapy for breast cancer on sexual function and health-related quality of life. Support Care Cancer [Internet]. 2016 jun [acesso em 2017 fev 17]; 24(6):2603-9. Disponivel em: http://link.springer.com/article/10.1007\%2Fs00520-015-3073-2

17. Santos LR, Tavares GB, Reis PED. Análise das respostas comportamentais ao câncer de mama utilizando o modelo adaptativo de Roy. Esc. Anna Nery [Internet]. 2012 set [acesso em 2016 ago 22]; 16(3): 459-65. Disponivel em: http://www.scielo.br/scielo.php?script=sci_arttextEpid=S141481452012000300005 \&lng=en 